\title{
Evaluation of endosonography in TN staging of oesophageal cancer
}

\author{
K Ziegler, C Sanft, M Zeitz, M Friedrich, H Stein, R Häring, E O Riecken
}

\begin{abstract}
Strategies for the treatment of cancer of the oesophagus depend on the tumour stage at the time of diagnosis. Resection, the only curative treatment, is confined to early tumour stages. Tumours with local infiltration are usually unresectable and require palliative treatment. Computed tomography has been widely used for preoperative staging but often fails to define this correctly. Endoscopic ultrasound allows direct visualisation of the parietal wall and may be useful in staging gastrointestinal tumours. In a comparative prospective study, 52 patients with tumours of the oesophagus were investigated preoperatively both by endoscopic ultrasound and computed tomography to determine the stage of tumour infiltration and local lymph node involvement. Thirty seven of these patients underwent operation, resection, or dissection and entered the study. The intraoperative findings or the histopathological assessment, or both, were taken as a reference. For all TN stages of oesophageal tumours, correct preoperative staging was accomplished by endoscopic ultrasound in $89 \%$ for $\mathbf{T}$ stage and $69 \%$ for $\mathbf{N}$ stage compared with $51 \%$ and $51 \%$ respectively by computed tomography (highly significant using Fisher's exact test). This study shows that endoscopic ultrasound is useful in preoperative TN staging of tumours of the oesophagus.
\end{abstract}

Patients with tumours of the oesophagus have a poor prognosis despite advances in diagnosis and treatment. In the Western world the five year survival has been reported to be from $4 \%$ to $10 \% .^{12}$

There are no characteristic symptoms in the early stages of these tumours. The mean period between the onset of symptoms and the final diagnosis is six months, by which time most tumours have reached an advanced stage. Resection of oesophageal tumours is the only curative treatment, but is limited to the early stages of disease, because tumours with local infiltration are usually unresectable. In these cases palliative treatments are available..$^{3-5}$ An exact staging of oesophageal tumours which includes information on depth of tumour infiltration and local and distant metastases is therefore of great importance if unnecessary operations are to be avoided.

Endoscopy of the oesophagus and the stomach with biopsy establishes the diagnosis and gives an orientating assessment of the tumour spread in the mucosa. The endoscopic findings are followed by $x$ ray examination of the oesophagus and the stomach. More detailed information on the extent and depth of tumour infiltration as well as the involvement of the lymph nodes is provided by computed tomography. In recent years, computed tomography has been reported to be reliable in staging oesophageal cancers, ${ }^{6-9}$ but several authors have recently claimed that it is inaccurate in defining local tumour infiltration and is therefore of little value in evaluating whether these tumours can be resected. ${ }^{10} 11$

A more recent method of examining the upper gastrointestinal tract is intraluminal endoscopically controlled ultrasound. ${ }^{12-16}$ In contrast to extracorporeal ultrasound, this form allows the use of higher sound frequencies with a higher resolution. Because of the close contact with the organs being studied, it is possible to examine the parietal wall in detail and to visualise its different layers. Moreover, a correlation has been shown in vitro between the echogenic layers and the histological layers of the parietal wall. ${ }^{17}$ This technique may therefore be able to visualise the depth of tumour infiltration. In addition, neighbouring organs can also be visualised with a high degree of resolution at a penetration depth of up to $8 \mathrm{~cm}$ and detection of local lymph node involvement seems to be possible. ${ }^{1618}$

We undertook a prospective study to determine whether endoscopic ultrasound is useful in the preoperative staging of tumours of the oesophagus. We found clear evidence that it is a more reliable clinical tool than computed tomography in TN staging of these tumours.

\section{Patients and methods}

PATIENTS

Between January 1986 and July 1988 all inpatients of the medical and surgical units of this hospital who were undergoing investigation of tumours of the oesophagus entered the study. A total of 52 patients was studied.

The 52 patients (median age: $57 \cdot 5$, range: 40 76 years; 15 women, 37 men) had histologically proved squamous cell carcinoma of the oesophagus. Four tumours were located in the upper third, 30 in the middle third, and 18 in the lower third of the oesophagus. Thirty four patients $(65 \%)$ underwent surgery. In 21 patients a subtotal oesophageal resection was performed; either transthoracic $(n=10)$ or transhiatal $(n=11)$. The tumour could not be resected in 13 patients (resection rate: $64 \%$ ).

In all patients both endoscopic ultrasound and computed tomography were carried out within 14 days of surgery.

Fifteen patients were not operated on because of their general clinical condition and distant metastases. A correlation between the endoscopic ultrasound scans and the computed tomograms 
TABLE I Patients with oesophageal tumours $(n=37)$ who underwent surgery. Results of preoperative staging by endoscopic ultrasound and computed tomography

\begin{tabular}{llll}
\hline & & & \\
Patient & Endoscopic ultrasound & Computed tomography & Intraoperative/pathological \\
findings
\end{tabular}

$\mathrm{T} 0=$ no tumour seen, $\mathrm{T} 1-4=$ depth of infiltration, $\mathrm{N} 0=$ no lymph node involvement, $\mathrm{N} 1=$ involvement of regional lymph nodes, ${ }^{\star}=$ tumour stenosis could not be passed.

was found in eight of these patients in respect of $\mathrm{T}$ status and in seven in respect of the $\mathrm{N}$ status.

Three patients with oesophageal cancer (Table I: patients 5, 19, and 34) were not operated on and died in hospital. The tumour stage of these patients was defined at necropsy. The necropsy examination was undertaken 32 days (range 1554 days) after endoscopic ultrasound and 36 days (range 17-60 days) after computed tomography.

The diagnostic accuracies in tumour staging of both endoscopic ultrasound and computed tomography were evaluated only in patients who underwent surgery. Thirty seven patients ful-

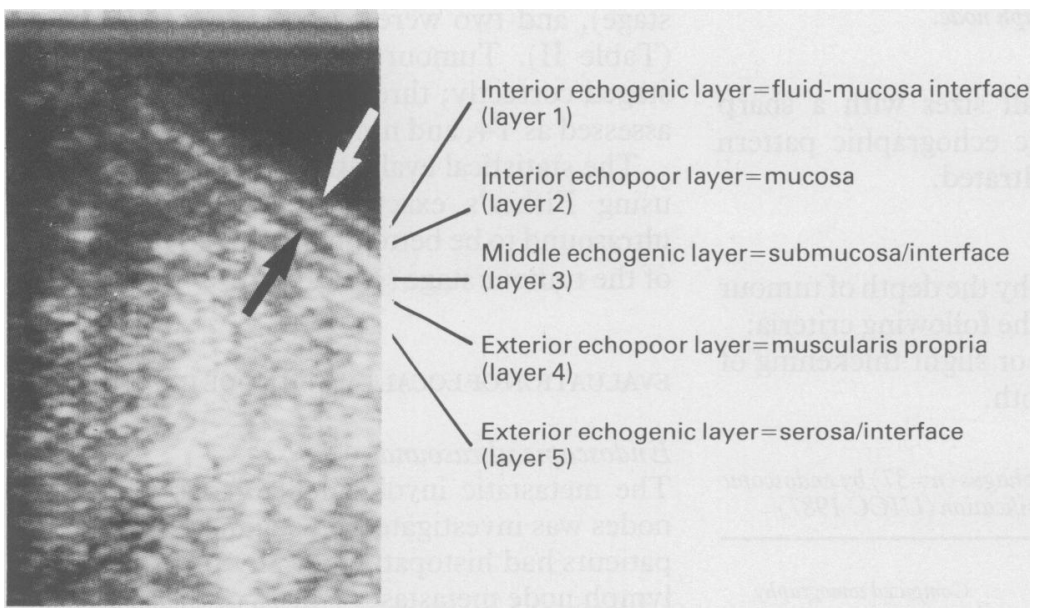

Figure 1: Endoscopic ultrasound showing normal oesophageal mucosa with the typical appearance of the five different layers and the relation between histological structures and echographic layers.

T1 stage = disturbance of echogenic layer 2/3, T2 stage=disturbance of echogenic layer 4 , T3 stage = disturbance of echogenic layer 5, T4 stage = tumour outside the echogenic layer 5 with signs of infiltrations. filled this criterion and the findings of both procedures were compared with the intraoperative findings or the histopathological assessment of the resected tumours, or both, or the results of dissections. The data of these patients are given in Table I

\section{ENDOSCOPIC ULTRASOUND}

Endoscopic ultrasound was performed by a fully trained endoscopist with a linear array scanner (Siemens Co, Erlangen, West Germany) (scanning ratio $1: 4$, scanning measure $4 \times 0 \cdot 192$, element distance $0.77 \mathrm{~mm}$, rated frequency $7 \cdot 5 \mathrm{MHz}$ ). The scanner was fixed to the tip of a Pentax FG 28 gastroscope (Hamburg, West Germany) and connected to the ultrasound unit (Sonoline 8000 from Siemens Co).

The patients were examined in a left lateral and semiupright position after premedication with intravenous diazepam (Valium, 5 to $10 \mathrm{mg}$ ). The ultrasonic endoscope was introduced under visual control and advanced to the stomach. To achieve good transmission the oesophagus was rinsed with water through the rinsing channel of the endoscope during the examination, which usually lasted 10 to 15 minutes. The ultrasonic visualisation of the gastro-oesophageal wall and its neighbouring structures was assessed by axial rotations of the endocscope in various sections. The findings were documented by polaroid camera and video tape recordings.

\section{COMPUTED TOMOGRAPHY}

Routinely performed computed tomography was carried out with the Somatom DRG or DRH from Siemens Co (section distance $8-10 \mathrm{~mm}$, scanning time 4.8 seconds). Some $150-250 \mathrm{ml}$ Ultraquist 300 (Schering AG, Berlin) intravenous contrast medium and $100-200 \mathrm{ml}$ of $1-2 \%$ oral Gastrografin (Schering AG, Berlin) were given to all patients.

\section{CLASSIFICATION OF THE TUMOUR STAGE}

The tumour stage was classified according to the TNM classification by the International Union Against Cancer Classification, $1987 .{ }^{19}$

\section{Endoscopic ultrasound}

With endoscopic ultrasound tumours were identified by thickening and disturbance of the five echogenic layers of the parietal wall (Fig 1). Depending on the depth of tumour infiltration into these layers, the $T$ stage was assessed as follows:

$\mathrm{T} 1=$ tumour has disturbed echogenic layers 1 and 2 (Fig 2A).

$\mathrm{T} 2=$ tumour has disturbed echogenic layers 1 to 3 (Fig 2B)

$\mathrm{T} 3=$ tumour has disturbed echogenic layers 1 to 5 .

T4=tumour has disturbed all echogenic layers 1 to 5 (Fig 2C) with infiltration of other organs.

The involvement of regional lymph nodes ( $\mathrm{N}$ stage) was shown by visualisation of perioesophageal and perigastric lymph nodes (Fig 


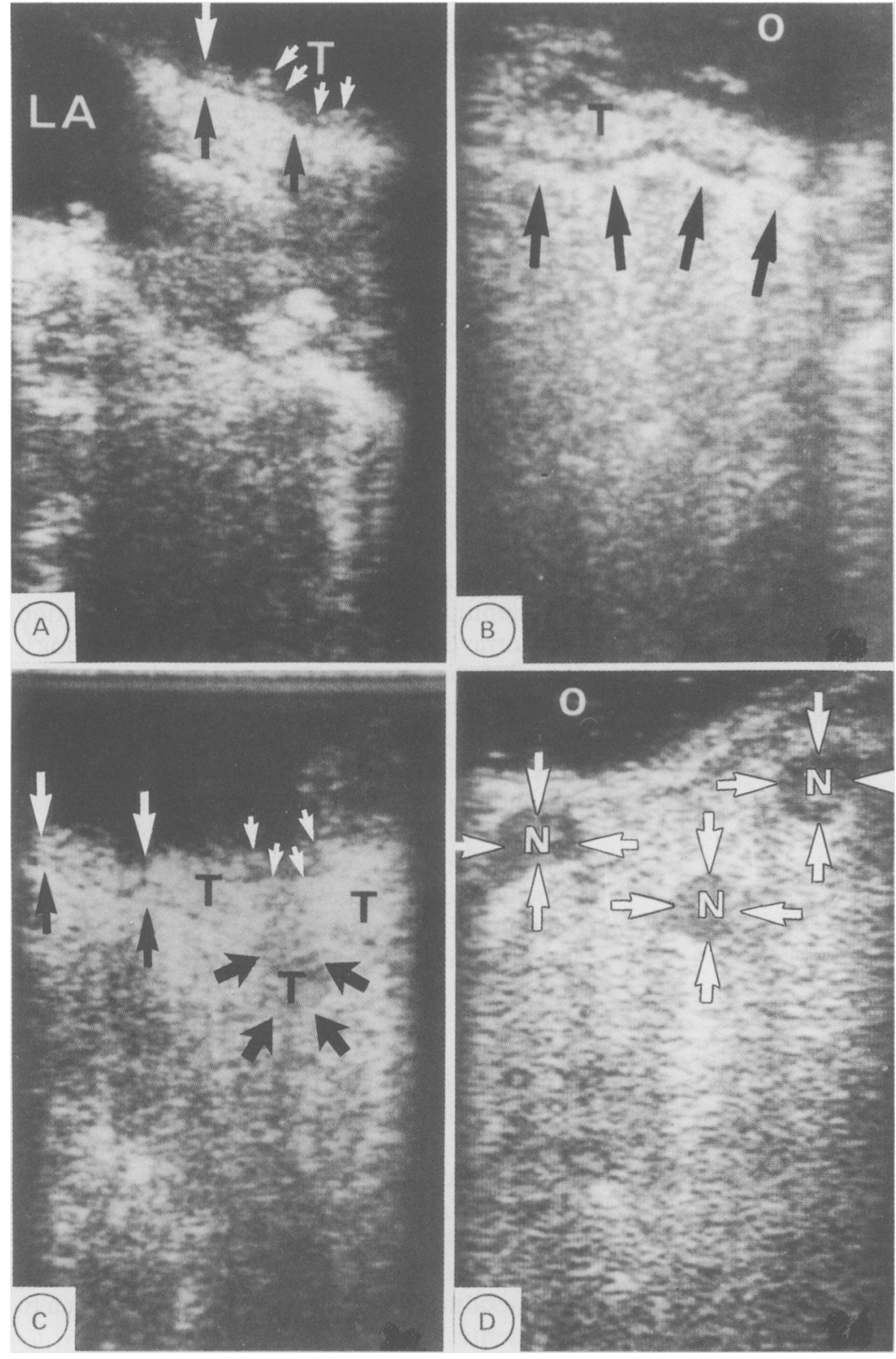

Figure 2: (A) T1 stage oesophageal cancer; the first hypoechoic layer representing the mucosa is disturbed (arrows).

(B) T2 stage oesophageal cancer; a disturbance of echogenic layers 1 to 3 can be seen showing infiltration of the muscularis propria of the oesophagus.

(C) Ulcerated tumour of the lower oesophagus (T4 stage); all echographic layers are disturbed and the tumour has infiltrated perioesophageal region (arrows).

(D) Perioesophageal lymph node metastasis. $N=$ infiltrated lymph node.

2D). Lymph nodes of all sizes with a sharp margin and a hypoechoic echographic pattern were considered to be infiltrated.

\section{Computed tomography}

With computed tomography the depth of tumour infiltration was based on the following criteria:

$\mathrm{T} 1=$ intraluminal mass or slight thickening of the wall ( 5 to $7 \mathrm{~mm})$, or both.

TABLE II Number (\%) of correct $T$ staging of tumours of the oesophagus $(n=37)$ by endoscopic ultrasound and computed tomography according to the TNM classification (UICC 1987)

\begin{tabular}{lccc}
\hline Stage & $\begin{array}{l}\text { Intraoperativel } \\
\text { histopathological assessment }\end{array}$ & Endoscopic ultrasound & Computed tomography \\
\hline T1 & 5 & $4(80 \%)$ & $1(20 \%)$ \\
T2 & 4 & $3(75 \%)$ & $7(58 \%)$ \\
T3 & 8 & $7(88 \%)$ & $11(55 \%)$ \\
T4 & 20 & $19(95 \%)$ & \\
\end{tabular}

$\mathrm{T} 2 / \mathrm{T} 3=$ thickening of the intestinal wall $(>7 \mathrm{~mm}<12 \mathrm{~mm})$ without evidence of local invasion.

T4= thickening of the wall with local invasion.

Lymph node metastases were defined by computed tomography as enlarged lymph nodes of more than $10 \mathrm{~mm}$ in diameter.

\section{STATISTICS}

The statistical significance of the data was evaluated by Fisher's exact test.

The study design was approved by the local ethics commission.

\section{Results}

\section{EVALUATION OF LOCAL TUMOUR GROWTH}

\section{Endoscopic ultrasound}

The individual data of patients with oesophageal tumours are given in Table I. Evaluation of the tumour stage by endoscopic ultrasound was possible in all cases. In seven patients the tumour stenosis could not be passed by the endosonoscope. In these patients only the proximal part of the tumour could be examined and tumour staging was therefore incomplete (Table I: patients $1,8,9,14,15,22$, and 23).

Carcinomas of the oesophagus restricted to the mucosa and submucosa $(n=5)$ could be staged correctly by endoscopic ultrasound as stage T1 (see Fig 2A) in four cases (Table II). Three of four T2 stages (see Fig 2B) were correctly staged, but one was overstaged (Table I: patient 3 ) as stage T3. Differentiation between T3 and T4 stage (see Fig 2C) oesophageal tumours $(n=28)$ was made by endoscopic ultrasound in all but two patients in whom the tumour was understaged (Table I: patients 1, and 30).

\section{Computed tomography}

Computed tomography was performed in all patients (Table I). Early oesophageal cancer $(n=5)$ was staged correctly as T1 in one patient, two had a normal computed tomogram (T0 stage), and two were overstaged as $\mathrm{T} 2 / 3$ or $\mathrm{T} 4$ (Table II). Tumours at stage $\mathrm{T} 2(\mathrm{n}=4)$ were staged correctly; three $\mathrm{T} 3$ staged tumours were assessed as T4, and nine T4 tumours as T2/3.

The statistical evaluation of the data obtained using Fisher's exact test showed endoscopic ultrasound to be better in preoperative definition of the tumour stage $(p=0 \cdot 000401)$.

\section{EVALUATION OF LOCAL LYMPH NODE INVOLVEMENT}

\section{Endoscopic ultrasound}

The metastatic involvement of regional lymph nodes was investigated ( $N$ staging). Twenty five patients had histopathologically proved regional lymph node metastases - in 16 of these, this was detected correctly by endoscopic ultrasound (Fig 2D, Table III). In the remaining patients the findings were false negative and in three of these perigastral lymph node metastasis could not be seen because of tumour stenosis (Table I: 
TABLE III Preoperative $N$ staging of tumours of the oesophagus $(n=37)$ using endoscopic ultrasound and computed tomography according to the TNM classification (UICC, 1987)

\begin{tabular}{llllll}
\hline & \multicolumn{2}{l}{ True } & & \multicolumn{2}{l}{ False } \\
\cline { 2 - 5 } Method & Positive & Negative & Positive & Negative \\
\hline Endoscopic ultrasound & 16 & 9 & 3 & 9 \\
Computed tomography & 10 & 9 & 4 & 14 \\
\hline
\end{tabular}

patients 1, 14, and 15). The absence of lymph node metastasis was classified correctly in nine of 12 patients. In three patients the findings were false positive.

\section{Computed tomography}

Computed tomography showed regional lymph node metastasis in 10 of 25 patients (Table III). In the remaining 15 the findings were false negative. No evidence of lymph node involvement was found correctly in nine of 12 tumours. The findings were false positive in four patients.

\section{Discussion}

This study shows that endoscopic ultrasound is useful in staging tumours of the oesophagus. With ultrasound it was possible to determine correctly the depth of tumour infiltration ( $\mathrm{T}$ stage) in $89 \%$ of patients. In contrast, computed tomography was able to define only $51 \%$ of tumours with regard to the $\mathrm{T}$ stage (Table II). The metastatic involvement of regional lymph nodes ( $\mathrm{N}$ stage) was depicted by endoscopic ultrasound with a higher specificity and sensitivity than computed tomography (Table III).

The different treatment strategies for oesophageal tumours should be determined by the patient's prognosis. ${ }^{20}$ The prognosis mainly results from the depth of tumour infiltration ( $T$ stage) and the involvement of local lymph nodes (N stage). Accurate preoperative information concerning continuous spread of the tumour into adjacent mediastinal structures and lymph node involvement (T4 N1 stage) could avoid unnecessary surgery and could identify those patients who would benefit more from palliative procedures such as local radiotherapy, ${ }^{5}$ laser therapy, ${ }^{3}$ oesophageal intubation, ${ }^{4}$ or chemotherapy. ${ }^{21}$

Computed tomography has been widely used in staging tumours of the upper intestinal tract. Its diagnostic accuracy, however, in evaluating oesophageal carcinoma is uncertain. There are a few studies that describe the possibility of defining different stages of tumour infiltration by computed tomography criteria. Some authors investigated the ability of computed tomography to predict whether oesophageal and cardiac tumours were operable ${ }^{7-922}$ by looking at local tumour infiltration. This seems to be possible because of the differentiation between soft tissue density tumours and mediastinal fat. ${ }^{23}$ On the other hand, computed tomography is not able to differentiate single layers of the intestinal wall. ${ }^{10}$ This limits its ability to separate early tumour stages (T1) from advanced stages (T2/T3).

In our study, computed tomography staged correctly $20 \%$ of early oesophageal tumours and $39-58 \%$ of advanced tumours (T2/3 stages). Recent studies have shown that local tumour infiltration can be predicted by computed tomography in approximately $50 \%$ of the cases. ${ }^{11}$ This is supported by the finding of a high rate of futile thoracotomies in these patients.

The clinical value of endoscopic ultrasound in our study is shown by its higher resolution and ability to differentiate between various histological structures of the intestinal wall using a linear array scanner that has high resolution in the near distance unlike sector scanners. The penetration depth of good resolution ultrasound is about $6-8 \mathrm{~cm}$, so the tumour and the adjacent organs can be visualised. In addition, it is not affected by pulsations. of the heart and the diaphragm, unlike computed tomography.

Advanced oesophageal tumours tend to be stenotic, preventing the passage of gastroscopes. In these cases endoscopic ultrasound can only visualise the proximal part of the tumour, meaning that staging is incomplete except for $\mathrm{T} 4, \mathrm{Nl}$ stages in the proximal part of the tumour. In our study, nine of 52 patients had tumour stenoses that could not be passed. Seven of these nine patients, however, underwent surgery and the tumour stage was defined. Four were found to have had their tumours staged correctly by endoscopic ultrasound.

In summary, our study shows that endoscopic ultrasound is better than computed tomography in staging the $\mathrm{T}$ and $\mathrm{N}$ status of oesophageal tumours. By introducing endoscopic ultrasound to the preoperative diagnostic programme, it may be possible to select more accurately patients with inoperable tumours. This method may therefore avoid unnecessary operations with potential risk for the patients. However, computed tomography, unlike endoscopic ultrasound, is able to visualise distant metastasis and should continue to be used for that purpose.

This work was supported by the Maria Sonnenfeld-Stiftung Part of this work has been presented at the Annual Meeting of the American Gastroenterological Association 1988 New Orleans.

1 Earlam R, Cunha-Melo JR. Oesophageal squamous cel carcinoma: I. A critical review of surgery. Br $\mathcal{F}$ Surg 1980 67: 381-90.

2 Giuli R, Gignoux M. Treatment of carcinoma of the esophagus. Ann Surg 1980; 192: 44-52.

3 Goldberg SJ, King K. Endoscopic Nd:YAG laser coagulation as palliative therapy for obstruction esophageal carcinoma. as palliative therapy for obstruction

4 Salo JA, Kalima TV. Fiber-endoscopic insertion of esophageal tube for palliation in malignant oesophagogastric obstruction. Acta Chir Scand 1985; 151: 341-4.

5 Rowland CG, Pagliero KM. Intracavitary irradiation in palliation of carcinoma of oesophagus and cardia. Lancet 1985; ii: 981-2.

6 Daffner RH, Halber MD, Postlethwait RW, Korobkin M, Thompson WM. CT of the esophagus. II. Carcinoma. $A \mathscr{F} R$ 1979; 133: 1051-5.

7 Moss AA, Schnyder P, Thoeni RF, Margulis AR. Esophageal carcinoma: pretherapy staging by computed tomography. carcinoma: pretherapy

8 Picus D, Balfe DM, Koehler RE, Roper CL, Owen JW. Computed tomography in the staging of esophageal

9 Thompson WM, Halvorsen RA, Foster WL Jr, Williford ME Postlethwait RW, Korobkin M. Computed tomography for
staging esophageal and gastroesophageal cancer: Reevaluastaging esophageal and gastroes

10 Quint LE, Glazer GM, Orringer MB, Gross BH. Esophageal carcinoma: CT findings. Radiology 1985; 155: 171-5.

11 Salonen O, Kivisaari L, Standertskjöld-Nordenstam C-G, Somer K, Virkkunen $\mathrm{P}_{\text {. }}$. Computed tomography in staging of esophageal carcinoma. Scand F Gastroenterol 1987; 22: 65-8.

12 Tio TL, den Hartog Jager FCA, Tijtgat GNJ. Endoscopic ultrasonography of non-Hodgkin lymphoma of the stomach. Gastroenterology 1986; 91: 401-8. 
13 Ziegler K, Sanft C, Semsch B, Gregor M, Friedrich M, Riecken EO. Prospektiv vergleichende Untersuchung zur Wertigkeit der Endosonographie für das präoperative Staging des Ösophaguskarzinoms. In: Henning H, Classen M. ing des Osophaguskarzinoms. In: Henning $\mathrm{H}$, Classen $\mathrm{M}$. Fortschritte der gastroenterologisch

14 Dancygier H, Classen M. Endosonographic diagnosis of benign pancreatic and biliary lesions. Scand $\mathcal{F}$ Gastroenterol 1986; 21 [suppl 123]: 119-22.

15 Caletti G, Bolondi L, Labò G. Anatomical aspects in ultrasonic endoscopy for the stomach. Scand $\mathcal{F}$ Gastroenterol 1984; 19 [suppl 94]: 34-42.

16 Aibe T, Ito T, Yoshida T, et al. Endoscopic ultrasonography of lymph nodes surrounding the upper GI tract. Scand $\mathcal{f}$

17 Tio TL, Tytgat NJ. Endoscopic ultrasonography of normal and pathologic upper gastrointestinal wall structure.
Comparison of studies in vivo and in vitro with histology. Scand 7 Gastroenterol $1986 ; 21$ [supply 123]: 27-33.

18 Polensky A, Ziegler K, Sanft C, Gregor M, Friedrich M, Stein
H, Riecken EO. Endosonographische Befunde benigner und maligner Läsionen der Magenwand. Prospektiver Vergleich mit herkömmlichen Untersuchungsverfahren. Disch Med Wochenschr 1988; 113: 1263-70.

19 International Union Against Cancer, TNM-Klassifikation maligner Tumoren. 4. Auflage, Berlin, Heidelberg, New

York: Springer, 1987.
Skinner DB, Little AG, Ferguson MK, Soriano A, Staszak VM. Selection of operation for esophageal cancer based on staging. Ann Surg 1986; 204: 391-400.

21 Kelsen DP, Hilaris E, Coonley C, Chapman R, Lesser M, Baens $M$. Cisplatin, vindesine and bleomycin chemotherapy
of regional and advanced esophageal cancer. $A m \mathcal{F} M e d 1983$; 75: 645-52.

22 Quint LE, Glazer GM, Orringer MB. Esophageal imaging by MR and CT: study of normal anatomy and neoplasms. Radiology 1985; 156: 727-31.

23 Freeny PC, Marks WM. Adenocarcinoma of the gastroesophageal junction: barium and CT examination. $A \mathscr{F} R$ esophageal junction: 